\title{
Poisson Reduction by Distributions
}

\author{
MADELEINE JOTZ and TUDOR S. RATIU \\ Section de Mathématiques and Bernoulli Center, Ecole Polytechnique Fédérale de Lausanne, \\ 1015 Lausanne, Switzerland.e-mail: madeleine.jotz@epfl.ch; tudor.ratiu@epfl.ch
}

Received: 3 December 2008 / Revised: 5 January 2009 / Accepted: 8 January 2009

Published online: 23 January 2009 - (C) Springer 2009

\begin{abstract}
It is shown that the singular Poisson reduction procedure can be improved for a large class of situations. In addition, Poisson reduction of orbit type manifolds is carried out in detail.
\end{abstract}

Mathematics Subject Classification (2000). 53D17， 37J15， 58A30.

Keywords. Poisson manifold, singular reduction, integrable distribution, proper action.

\section{Introduction}

A smooth Poisson manifold $(M,\{\cdot, \cdot\})$ with a free and proper action of a Lie group $G$ on it can be reduced and the quotient space inherits a Poisson structure induced from the one on $M$. The reduced space can be seen as the leaf space of the vertical distribution of the action, that is, the distribution spanned by the fundamental vector fields $\xi_{M}, \xi \in \mathfrak{g}$ (where $\mathfrak{g}$ is the Lie algebra of $G$ ).

This leads to the approach of Marsden and Ratiu [3] of Poisson reduction by distributions: one is given an embedded submanifold $S$ of $M$ and a vector bundle $\left.B \subset T M\right|_{S}$ such that $B_{S}=B \cap T S$ is a canonical regular integrable distribution on $S$. Then there is a necessary and sufficient condition for the leaf space $S / B_{S}$ to inherit a smooth Poisson structure from the one on $M$. It was shown in Falceto and Zambon [1] that this necessary and sufficient condition always holds unless the distribution $B$ is zero.

The method of Marsden and Ratiu [3] was generalized in Ortega and Ratiu [5] to an arbitrary decomposed subset $S$ of $M$ and distributions in $\left.T M\right|_{S}$ adapted to the decomposition of $S$ (see also Ortega and Ratiu [4]). This raises the following question: does an analogous statement to the results of Falceto and Zambon [1] hold for the generalized situation of Ortega and Ratiu [5]? In this note we present a result that not only recovers the statement in Falceto and Zambon [1] but is also applicable to the singular situation.

Partially supported by a Swiss NSF grant. 
In addition, we show that if a Lie group acts properly and canonically on a Poisson manifold, each stratum of the reduced space inherits a Poisson structure; the necessary and sufficient condition for the generalized Reduction Theorem for Poisson Manifolds by Pseudogroups is always satisfied if the considered pseudogroup is a Lie group and the subset of $M$ an orbit type of the action (hence an embedded submanifold).

All definitions and notations needed here can be found in Ortega and Ratiu [4]. The statements used in this note without proof are also taken from this book. We will specify at the beginning of each section which section of this book is used.

\section{The Reduction Theorem}

\subsection{DEFINITIONS AND NOTATIONS}

The following background material is taken from Ortega and Ratiu [4, Section 10.4].

Let $M$ be a differentiable manifold and $S \subseteq M$ a decomposed subset of $M$. Let $\left\{S_{i}\right\}_{i \in I}$ be the pieces of this decomposition. The topology of $S$ is not necessarily the relative topology as a subset of $M$. We say that $\left.D \subset T M\right|_{S}$ is a smooth generalized distribution (that is, not necessarily of constant rank) on $S$ adapted to the decomposition $\left\{S_{i}\right\}_{i \in I}$ if $D_{S_{i}}:=D \cap T S_{i}$ is a smooth distribution on $S_{i}$ for all $i \in I$.

The distribution is said to be integrable if $D_{S_{i}}$ is integrable for each $i \in I$. Then, we can partition each $S_{i}$ into the corresponding maximal integral manifolds. The resulting equivalence relations on each $S_{i}$ induce an equivalence relation $D_{S}$ on the whole set $S$. Define

$$
S / D_{S}:=\cup_{i \in I} S_{i} / D_{S_{i}}
$$

and let $\pi_{D_{S}}: S \rightarrow S / D_{S}$ be the natural projection. We say that a subset $R \subset S$ is $D_{S}$-invariant if it is invariant under the flow of any section of $D_{S_{i}}$, for all $i \in I$.

We define the presheaf of smooth functions $C_{S / D_{S}}^{\infty}$ on $S / D_{S}$ as the map that associates to any open subset $V$ of $S / D_{S}$ the set of functions $C_{S / D_{S}}^{\infty}(V)$ characterized by the following property: $f \in C_{S / D_{S}}^{\infty}(V)$ if and only if for any $z \in V$ there exists $m \in \pi_{D_{S}}^{-1}(z), U_{m}$ an open neighborhood of $m \in M$ such that $U_{m} \cap S$ is $D_{S}$-invariant, and $F \in C_{M}^{\infty}\left(U_{m}\right)$ satisfying

$$
\left.f \circ \pi_{D_{S}}\right|_{\pi^{-1}(V) \cap U_{m}}=\left.F\right|_{\pi^{-1}(V) \cap U_{m}} .
$$

We say that $F$ is a local extension of $f \circ \pi_{D_{S}}$ at the point $m \in \pi_{D_{S}}^{-1}(V)$.

Recall the following definitions.

DEFINITION 2.1. Let $(M,\{\cdot, \cdot\})$ be a Poisson manifold, $S \subset M$ a decomposed space, and $\left.D \subset T M\right|_{S}$ a smooth distribution adapted to the decomposition $\left\{S_{i}\right\}_{i \in I}$ of $S$. The distribution $D$ is called Poisson or canonical if the condition $\left.\mathbf{d} f\right|_{D}=$ $\left.\mathbf{d} g\right|_{D}=0$, for any $f, g \in C_{M}^{\infty}(U), U \subset M$ an open subset, implies that $\left.\mathbf{d}\{f, g\}\right|_{D}=0$. 
DEFINITION 2.2. The presheaf $C_{S / D_{S}}^{\infty}$ is said to have the $\left(D, D_{S}\right)$-local extension property when the topology of $S$ is stronger than the relative topology and the local extensions of $f \circ \pi_{D_{S}}$ defined in (1) can always be chosen to satisfy

$$
\left.\mathbf{d} F(n)\right|_{D(n)}=0, \quad \text { for any } \quad n \in \pi_{D S}^{-1}(V) \cap U_{m} .
$$

We say that $F$ is a local $D$-invariant extension of $f \circ \pi_{D_{S}}$ at the point $m \in \pi_{D_{S}}^{-1}(V)$.

We immediately get the following result.

LEMMA 2.3. Let $D$ be canonical and $C_{S / D_{S}}^{\infty}$ have the $\left(D, D_{S}\right)$-local extension property. If $F$ and $G \in C_{M}^{\infty}\left(U_{m}\right)$ are local $D$-invariant extensions of $f \circ \pi_{D_{S}}$ and $g \circ \pi_{D_{S}}$ at $m \in \pi_{D_{S}}^{-1}(V)$, then their Poisson bracket $\{F, G\}$ is also local D-invariant.

This statement is needed in the proof of the reduction theorem (Theorem 10.4.8 in Ortega and Ratiu [4]). Note that if $S$ is an embedded submanifold of $M$ and $\left.D \subseteq T M\right|_{S}$ a subbundle of the tangent bundle of $M$ restricted to $S$ such that $D_{S}:=$ $D \cap T S$ is a smooth, integrable subbundle of $T S$, the distribution $D$ is canonical in the sense of Definition 2.1 if and only if it is canonical in the sense of Marsden and Ratiu [3] and Falceto and Zambon [1].

\subsection{THE REDUCTION THEOREM}

We present here a proof of the Poisson Reduction by Distributions Theorem in Ortega and Ratiu [4]. This proof is not new but since it is only outlined in Ortega and Ratiu [4], we decided to present it here to better put in context the proposition following it.

DEFINITION 2.4. Let $(M,\{\cdot, \cdot\})$ be a Poisson manifold, $S$ a decomposed subset of $M$, and $\left.D \subset T M\right|_{S}$ a Poisson integrable distribution that is adapted to the decomposition of $S$. Assume that $C_{S / D_{S}}^{\infty}$ has the $\left(D, D_{S}\right)$-local extension property. We say that $(M,\{\cdot, \cdot\}, D, S)$ is Poisson reducible when $\left(S / D_{S}, C_{S / D_{S}}^{\infty},\{\cdot, \cdot\}^{S / D_{S}}\right)$ is a well-defined presheaf of Poisson algebras where, for any open set $V \subset S / D_{S}$, the bracket

$$
\{\cdot, \cdot\}_{V}^{S / D_{S}}: C_{S / D_{S}}^{\infty}(V) \times C_{S / D_{S}}^{\infty}(V) \rightarrow C_{S / D_{S}}^{\infty}(V)
$$

is given by

$$
\{f, g\}_{V}^{S / D_{S}}\left(\pi_{D_{S}}(m)\right):=\{F, G\}(m),
$$

for any $m \in \pi_{D_{S}}^{-1}(V)$ and local $D$-invariant extensions $F$ and $G$ at $m$ of $f \circ \pi_{D_{S}}$ and $g \circ \pi_{D_{S}}$, respectively. 
THEOREM 2.5. Let $(M,\{\cdot, \cdot\})$ be a Poisson manifold with associated Poisson bivector field $B \in \Gamma\left(\Lambda^{2}(T M)\right), S$ a decomposed space, and $\left.D \subset T M\right|_{S}$ a Poisson integrable generalized distribution adapted to the decomposition of $S$. Assume that $C_{S / D_{S}}^{\infty}$ has the $\left(D, D_{S}\right)$-local extension property. Then $(M,\{\cdot, \cdot\}, D, S)$ is Poisson reducible if and only if for any $m \in S$

$$
B^{\sharp}\left(\Delta_{m}\right) \subset\left(\Delta_{m}^{S}\right)^{\circ}
$$

where

$$
\Delta_{m}:=\left\{\begin{array}{l|l}
\mathbf{d} F(m) & \begin{array}{l}
F \in C_{M}^{\infty}\left(U_{m}\right),\left.\mathbf{d} F(s)\right|_{D(s)}=0 \text { for all } s \in U_{m} \cap S, \\
\text { and for any open neighborhood } U_{m} \text { of } m \text { in } M
\end{array}
\end{array}\right\},
$$

and

$$
\Delta_{m}^{S}:=\left\{\begin{array}{l|l}
\mathbf{d} F(m) \in \Delta_{m} & \begin{array}{l}
\left.F\right|_{U_{m} \cap V_{m}} \text { is constant for an open neighborhood } U_{m} \\
\text { of } m \text { in } M \text { and an open neighborhood } V_{m} \text { of } m \text { in } S
\end{array}
\end{array}\right\} .
$$

Remark 2.6. If $S$ is endowed with the relative topology, then the definition of $\Delta_{m}^{S}$ simplifies to

$$
\Delta_{m}^{S}=\left\{\begin{array}{l|l}
\mathbf{d} F(m) \in \Delta_{m} & \begin{array}{c}
\left.F\right|_{U_{m} \cap S} \text { is constant for an open } \\
\text { neighborhood } U_{m} \text { of } m \text { in } M
\end{array}
\end{array}\right\} .
$$

This observation will be crucial in the proof of the next proposition.

Proof. Assume that $(M,\{\cdot, \cdot\}, D, S)$ is Poisson reducible. Let $m \in S$ and choose $\mathbf{d} F(m) \in \Delta_{m}$, that is, $F \in C_{M}^{\infty}\left(U_{m}\right)$ where $U_{m}$ is an open neighborhood of $m$ in $M$, such that $U_{m} \cap S$ is $D_{S}$-invariant and $\left.\mathbf{d} F(s)\right|_{D(s)}=0$ for all $s \in U_{m} \cap S$. Choose in the same manner an arbitrary $\mathbf{d} G(m) \in \Delta_{m}^{S}$, that is, $G \in C_{M}^{\infty}\left(U_{m}\right)$, such that $\left.\mathbf{d} G(s)\right|_{D(s)}=$ 0 for all $s \in U_{m} \cap S$ and $\left.G\right|_{U_{m} \cap V_{m}}=k \in \mathbb{R}$ is constant, where $V_{m}$ is an open neighborhood of $m$ in $S$. Since $\left.F\right|_{S \cap U_{m}}$ and $\left.G\right|_{S \cap U_{m}}$ are $D_{S}$-invariant, they have constant values on the leaves of $D_{S}$. Hence, if $V \subset S / D_{S}$ is the open set $V:=\pi_{D_{S}}\left(U_{m} \cap S\right)$ (note that $U_{m} \cap S$ is open in $S$ because the topology of $S$ is stronger than the topology induced from $M$ ), the functions $F, G \in C_{M}^{\infty}\left(U_{m}\right)$ define $f, g \in C_{S / D_{S}}^{\infty}(V)$ such that $\left.f \circ \pi_{D_{S}}\right|_{\pi_{D_{S}}^{-1}(V) \cap U_{m}}=\left.F\right|_{\pi_{D_{S}}^{-1}(V) \cap U_{m}}$ and $\left.g \circ \pi_{D_{S}}\right|_{\pi_{D_{S}}^{-1}(V) \cap U_{m}}=\left.G\right|_{\pi_{D_{S}}^{-1}(V) \cap U_{m}}$. Since $G$ is constant on $U_{m} \cap V_{m}$, the function $g$ is constant on the neighborhood $\pi_{D_{S}}\left(U_{m} \cap V_{m}\right)$ of $\pi_{D_{S}}(m)$ in $S / D_{S}$ and thus we have

$$
\{f, g\}_{V}^{S / D_{S}}\left(\pi_{D_{S}}(m)\right)=0 .
$$

This yields $\{F, G\}(m)=\{f, g\}_{V}^{S / D_{S}}\left(\pi_{D_{S}}(m)\right)=0$ and hence

$$
\mathbf{d} G(m)\left(B^{\sharp}(\mathbf{d} F(m))\right)=\{F, G\}(m)=0 .
$$

This proves the desired inclusion

$$
B^{\sharp}\left(\Delta_{m}\right) \subset\left(\Delta_{m}^{S}\right)^{\circ} .
$$


Conversely, assume that (2) is satisfied for all $m \in S$. We show that $(M,\{\cdot, \cdot\}, D, S)$ is Poisson reducible, hence, that $\left(S / D_{S}, C_{S / D_{S}}^{\infty},\{\cdot, \cdot\}^{S / D_{S}}\right)$ given in Definition 2.4 is well-defined. Recall the definition of $\{\cdot, \cdot\}^{S / D_{S}}$ : if $V$ is an open subset of $S / D_{S}$ and $f, g \in C_{S / D_{S}}^{\infty}(V)$, then for all $m \in \pi_{D_{S}}^{-1}(V)$, we define $\{f, g\}_{V}^{S / D_{S}}\left(\pi_{D_{S}}(m)\right)=\{F, G\}(m)$, where $F, G \in C_{M}^{\infty}\left(U_{m}\right)$ are local $D$-invariant extensions of $f \circ \pi_{D_{S}}$ and $g \circ \pi_{D_{S}}$, respectively, and $U_{m}$ is an open neighborhood of $m \in M$ such that $U_{m} \cap S$ is $D_{S}$-invariant.

We want to show that the following definition of $\{f, g\}_{V}^{S / D_{S}} \in C_{S / D_{S}}^{\infty}(V)$ makes sense:

$$
\left.\{f, g\}_{V}^{S / D_{S}} \circ \pi_{D_{S}}\right|_{U_{m} \cap \pi_{D_{S}}^{-1}(V)}:=\left.\{F, G\}\right|_{U_{m} \cap \pi_{D_{S}}^{-1}(V)} .
$$

Since $D$ is canonical, by Lemma 2.3 , the function $\left.\{F, G\}\right|_{U_{m} \cap S}$ is $D$-invariant and thus constant along the integral curves of any section of $D_{S_{i}}$ at points where this makes sense. Let $m, m^{\prime} \in M$ be such that $\pi(m)=\pi\left(m^{\prime}\right)$. Let $F^{\prime}, G^{\prime} \in C_{M}^{\infty}\left(U_{m^{\prime}}\right)$ be local $D$-invariant extensions of $f \circ \pi_{D_{S}}$ and $g \circ \pi_{D_{S}}$, respectively, where $U_{m^{\prime}}$ is an open neighborhood of $m^{\prime} \in M$ such that $U_{m^{\prime}} \cap S$ is $D_{S}$-invariant. Let $i_{0} \in I$ be the index such that $m \in S_{i_{0}}$. Then, by definition of $\pi_{D_{S}}, m^{\prime} \in S_{i_{0}}$ can be connected to $m$ by a finite union of integral curves of sections of $D_{S_{i_{0}}}$ and hence $\left\{F^{\prime}, G^{\prime}\right\}(m)=\left\{F^{\prime}, G^{\prime}\right\}\left(m^{\prime}\right)$. Since $U_{m} \cap S$ and $U_{m^{\prime}} \cap S$ are both $D_{S}$-invariant, it follows that $U_{m} \cap U_{m^{\prime}} \cap S$ is also $D_{S}$-invariant and contains $m$ and $m^{\prime}$. Thus, we have only to check that $\left\{F^{\prime}, G^{\prime}\right\}(m)=\{F, G\}(m)$, that is, that $\{F, G\}(m)$ does not depend on the choice of the extensions $F$ and $G$. Because of the antisymmetry of $\{\cdot, \cdot\}$, it suffices to show that it does not depend on the choice of the extension $G$. Let $G^{\prime} \in C_{M}^{\infty}\left(U_{m}\right)$ be another local $D$-invariant extension of $g \circ \pi_{D_{S}}$. Then we have $\left.\left(G-G^{\prime}\right)\right|_{U_{m} \cap \pi^{-1}(V)}=0$ and $\left.\mathbf{d}\left(G-G^{\prime}\right)\right|_{D(s)}=\left.\mathbf{d} G\right|_{D(s)}-\left.\mathbf{d} G^{\prime}\right|_{D(s)}=0$ for all $s \in U_{m} \cap S$. Hence $\mathbf{d}\left(G-G^{\prime}\right)(m) \in \Delta_{m}^{S}$. Since $\mathbf{d} F(m) \in \Delta_{m}$ by definition, we get using (2):

$$
\left\{F, G-G^{\prime}\right\}(m)=\mathbf{d}\left(G-G^{\prime}\right)(m)\left(B^{\sharp}(\mathbf{d} F(m))\right)=0 .
$$

This yields $\{F, G\}(m)=\left\{F, G^{\prime}\right\}(m)$.

The Leibniz and Jacobi identities for $\{\cdot, \cdot\}_{V}^{S / D_{S}}$ follow directly from the definition of $\{\cdot, \cdot\}_{V}^{S / D_{S}}$ and the fact that $\{\cdot, \cdot\}$ satisfies these identities.

If $S$ is a submanifold of $M$ and $D$ is a subbundle of $\left.T M\right|_{S}$ such that $D_{S}=$ $D \cap T S$ is a smooth regular integrable subbundle of $T S$, we have $\Delta_{m}=D(m)^{\circ}$ and $\left(\Delta_{m}^{S}\right)^{\circ}=T_{m} S+D(m)$ (note that all annihilators are pointwise annihilators). In this setting, a lemma in Falceto and Zambon [1] shows that if $D$ is canonical, then it is either trivial or $B^{\sharp}\left(D(m)^{\circ}\right)=B^{\sharp}\left(\Delta_{m}\right)$ is automatically contained in $T_{m} S \subseteq\left(\Delta_{m}^{S}\right)^{\circ}$.

The following is a direct generalization of this lemma in Falceto and Zambon [1].

PROPOSITION 2.7. In the setting of Theorem 2.5, if the topology of $S$ coincides with the relative topology of $S$ as a subset of $M$ and if $D$ does not vanish (at any 
point) on $S$, we have $B^{\sharp}\left(\Delta_{m}\right) \subset\left(\Delta_{m}^{S}\right)^{\circ}$ for all $m \in S$. Thus, if $S$ is endowed with the relative topology, condition (2) in Theorem 2.5 automatically holds.

Proof. Assume that there exists $m \in S$ such that $B^{\sharp}\left(\Delta_{m}\right) \not \subset\left(\Delta_{m}^{S}\right)^{\circ}$. Then we find $F, G \in C_{M}^{\infty}\left(U_{m}\right)$, where $U_{m}$ is an open neighborhood of $m$ in $M$, such that $\left.\mathbf{d} F(s)\right|_{D(s)}=\left.\mathbf{d} G\right|_{D(s)}=0$ for all $s \in U_{m} \cap S$, and $\left.G\right|_{U_{m} \cap S}=k \in \mathbb{R}$ is constant, such that $\{F, G\}(m)=\mathbf{d} G(m)\left(B^{\sharp}(\mathbf{d} F(m))\right) \neq 0$. Note that we have used here in an essential way the fact that $S$ is endowed with the relative topology; see Remark 2.6. Without loss of generality, we have $\left.G\right|_{U_{m} \cap S}=0$ (otherwise, replace $G$ with $G-k$ ).

Let $H \in C_{M}^{\infty}\left(U_{m}^{\prime}\right)$ be an arbitrary function defined on a neighborhood $U_{m}^{\prime} \subset U_{m}$ of $m \in M$. Then we have $\left.(H G)\right|_{U_{m}^{\prime} \cap S}=0$ and $\left.\mathbf{d}(H G)(s)\right|_{D(s)}=\left.G(s) \mathbf{d} H(s)\right|_{D(s)}+$ $\left.H(s) \mathbf{d} G\right|_{D(s)}=0$ for all $s \in U_{m}^{\prime} \cap S$. Thus, we get $\left.\mathbf{d}(H G)\right|_{D}=0$ on $U_{m}^{\prime}$. Since $\left.\mathbf{d} F\right|_{D}=$ 0 on $U_{m}^{\prime}$ by construction, we get $\left.\mathbf{d}\{F, G H\}\right|_{D}=0$ on $U_{m}^{\prime}$ because $D$ is canonical. This yields:

$$
\begin{aligned}
0= & \left.\mathbf{d}\{F, G H\}\right|_{D(m)}=\left.\mathbf{d}(G\{F, H\}+H\{F, G\})\right|_{D(m)}= \\
= & \left.G(m) \mathbf{d}\{F, H\}\right|_{D(m)}+\left.\{F, H\}(m) \mathbf{d} G\right|_{D(m)}+ \\
& +\left.H(m) \mathbf{d}\{F, G\}\right|_{D(m)}+\left.\{F, G\}(m) \mathbf{d} H\right|_{D(m)}= \\
= & \left.0 \cdot \mathbf{d}\{F, H\}\right|_{D(m)}+\{F, H\}(m) \cdot 0+H(m) \cdot 0+\left.\{F, G\}(m) \mathbf{d} H\right|_{D(m)}= \\
= & \left.\{F, G\}(m) \mathbf{d} H\right|_{D(m)} .
\end{aligned}
$$

Thus, we get $\left.\mathbf{d} H\right|_{D(m)}=0$. Because $H$ was arbitrary, we have $\alpha_{m}(D(m))=0$ for all $\alpha_{m} \in T_{m}^{*} M$. Hence we get $D(m)=0$, a contradiction since $D$ does not vanish on $S$ by hypothesis.

\section{Poisson Reduction by Proper Symmetries}

In this section we use Ortega and Ratiu [4, Section 10.2].

Let $G$ be a Lie group acting properly and canonically on the Poisson manifold $M$. Let $S$ be an orbit type manifold of the action. Proposition 2.7 cannot be applied if we take $D$ to be the restriction $\mathcal{V}$ of the vertical distribution $\operatorname{span}\left\{\xi_{M} \mid\right.$ $\xi \in \mathfrak{g}$ \} to $S$ since $\mathcal{V}$ can vanish on $S$. In spite of this, we know from Jotz et al. [2] that $(M,\{\cdot, \cdot\}, \mathcal{V}, S)$ is always reducible. This was done using singular Dirac reduction. We will prove this result here directly.

To do this, it is more natural to show that the hypotheses for the Poisson Reduction by Pseudogroups (see Ortega and Ratiu [4]) are always satisfied if the pseudogroup is the group of diffeomorphisms associated to the proper and canonical action of a Lie group $G$ on $M$. The Poisson reduction of $S$ by the vertical space $\mathcal{V}$ of the action is then the counterpart of this theorem in the approach of Poisson reduction by distributions.

Let, in general, $(M,\{\cdot, \cdot\})$ be a smooth Poisson manifold, $G$ a Lie group acting properly and canonically on $M$, and $S \subset M$ an embedded $G$-invariant submanifold of $M$. Then $G$ acts properly on $S$ and we can regard the quotient $S / G$ as a subset 
of $M / G$. Let $\pi: M \rightarrow M / G$ be the projection and $C^{\infty}(M / G):=C_{M, G}^{\infty}(M / G)$ be the set of functions $\bar{f}$ on $M / G$ such that $\pi^{*} \bar{f} \in C^{\infty}(M)^{G}$ (we denote by $C^{\infty}(M)^{G}$ the $G$-invariant smooth functions on $M$ ). There is a well-defined presheaf of Whitney smooth functions $C_{M / G}^{\infty}(S / G)$ on $S / G$ induced by $C^{\infty}(M / G)$, that is, for $\tilde{f} \in$ $C_{M / G}^{\infty}(S / G)$ defined on the open set $V \subseteq S / G$, there exists $\bar{f} \in C^{\infty}(M / G)$ defined on the open set $U \subseteq M / G$ such that $\left.\tilde{f}\right|_{U \cap V}=\left.\bar{f}\right|_{U \cap V}$. Properness of the action and the fact that $S$ is a $G$-invariant embedded submanifold of $M$ yield the equality

$$
C_{M / G}^{\infty}(S / G)=C_{S, G}^{\infty}(S / G) \text {. }
$$

DEFINITION 3.1. We say that $(M,\{\cdot, \cdot\}, G, S)$ is Poisson reducible when

$$
\left(S / G, C_{M / G}^{\infty}(S / G),\{\cdot, \cdot\}^{S / G}\right)
$$

is a well-defined presheaf of Poisson algebras where, for any open set $V \subset S / G$, the bracket $\{\cdot, \cdot\}_{V}^{S / G}: C_{M / G}^{\infty}(V) \times C_{M / G}^{\infty}(V) \rightarrow C_{M / G}^{\infty}(V)$ is given by

$$
\{f, h\}_{V}^{S / G}\left(\pi_{S}(m)\right)=\{F, H\}(m)
$$

for any $m \in \pi_{S}^{-1}(V)$ and any $G$-invariant local extensions $F, H \in C^{\infty}(M)^{G}$ at $m$ of $f \circ \pi_{S}$ and $h \circ \pi_{S}$, respectively.

We will show that the necessary condition of the following theorem is always satisfied if $S$ is an orbit type manifold of the proper action of $G$ on $M$.

THEOREM 3.2. Let $G$ be a Lie group acting properly and canonically on the Poisson manifold $(M,\{\cdot, \cdot\})$. Let $S$ be an embedded G-invariant submanifold of $M$. Then $(M,\{\cdot, \cdot\}, G, S)$ is Poisson reducible if

$$
B^{\sharp}\left(\left((\mathfrak{g} \cdot m)^{\circ}\right)^{G_{m}}\right) \subset T_{m} S, \quad \text { for any } m \in S .
$$

Note that $\left((\mathfrak{g} \cdot m)^{\circ}\right)^{G_{m}}$ is equal to

$$
\operatorname{span}\left\{\mathbf{d} f(m) \mid f \in C^{\infty}(M)^{G} \text { defined on a neighborhood of } m\right\} .
$$

We recall here some facts about proper actions. Let $\Phi: G \times M \rightarrow M$ be a proper action of the connected Lie group $G$ on $M$. Define for each compact subgroup $H$ of $G$ the orbit type manifold

$$
M_{(H)}=\left\{m \in M \mid G_{m} \text { is conjugated to } H\right\},
$$

and the isotropy type manifold

$$
M_{H}=\left\{m \in M \mid G_{m}=H\right\},
$$

where $G_{m}$ is the isotropy subgroup of the point $m \in M$. Connected components of $M_{(H)}$ and $M_{H}$ are embedded submanifolds of $M$. The connected components of the orbit type manifolds form a Whitney stratification of the manifold $M$. 
The quotient space $\bar{M}:=M / G=\{G m \mid m \in M\}$ is also a Whitney stratified space. Its strata are the connected components of the sets $\pi\left(M_{(H)}\right)=\pi\left(M_{H}\right)$, where $\pi$ : $M \rightarrow \bar{M}$ is the projection. Let $P$ be a connected component of an orbit type manifold $M_{(H)}$. The subgroup $G^{P}:=\left\{g \in G \mid \Phi_{g}(P) \subseteq P\right\} \subset G$ is a union of connected components of $G$ and is hence a Lie subgroup of $G$. Indeed, it is clear that the connected component of the identity $G^{\circ}$ of $G$ is a subgroup of $G^{P}$. The connected component of any other element $g \in G$ equals $g G^{\circ}$. For any $h \in G^{\circ}$ and $g \in G^{P}$, we have $\Phi_{g h}(P)=\Phi_{g}\left(\Phi_{h}(P)\right) \subseteq \Phi_{g}(P) \subseteq P$, which shows that $g G^{\circ} \subseteq G^{P}$.

Hence the proper action of $G$ on $M$ restricts to a proper action $\Phi^{P}$ of $G^{P}$ on $P$ satisfying $\iota_{P} \circ \Phi_{g}^{P}=\Phi_{g} \circ \iota_{P}$ for all $g \in G^{P}$. Moreover, the action $\Phi^{P}$ has conjugated isotropy subgroups and thus the quotient $P / G^{P}$ is a smooth manifold. Let $\pi_{P}: P \rightarrow P / G^{P}$ be the quotient map. The quotient $P / G^{P}$ is diffeomorphic to the stratum $\pi(P)=\bar{P}$ of $\bar{M}$, that is, the differentiable structures on $\bar{P}$ as a subset of $\bar{M}$ and as the quotient of $P$ by the action of $G^{P}$ coincide. The proof of this can be found, for example, in Jotz et al. [2].

The smooth generalized distribution $\mathcal{T}$ spanned by the smooth $G$-invariant vector fields on $M$ is integrable in the sense of Sussmann. Its leaves are the connected components of the isotropy type manifolds, that is, the $G$-invariant vector fields on $M$ are tangent to the isotropy type manifolds (and hence to the orbit type manifolds); see Ortega and Ratiu [4, Section 3.5] for a proof of this fact.

PROPOSITION 3.3. Let $(M,\{\cdot, \cdot\})$ be a smooth Poisson manifold and $G$ a Lie group acting properly and canonically on $(M,\{\cdot, \cdot\})$. Let $S:=\pi^{-1}(\bar{S})$, where $\bar{S}$ is a stratum of $\bar{M}$. Then, for any $m \in S$, the following inclusion holds:

$$
B^{\sharp}\left(\left((\mathfrak{g} \cdot m)^{\circ}\right)^{G_{m}}\right) \subset T_{m} S .
$$

Therefore, $(M,\{\cdot, \cdot\}, G, S)$ is Poisson reducible.

Proof. Note that $S$ is a union of connected components having the same dimension of some orbit type manifold. Hence $S$ is a $G$-invariant submanifold of $M$.

Let $m \in S$ and $f$ a $G$-invariant function defined on a neighborhood of $m$. Since the action of $G$ on $M$ is canonical, we have for all $g \in G$ and all $h \in C^{\infty}(M)$

$$
\begin{aligned}
\Phi_{g}^{*}\left(B^{\sharp}(\mathbf{d} f)\right)(\mathbf{d} h) & =\mathbf{d} h\left(T \Phi_{g^{-1}} \circ B^{\sharp}(\mathbf{d} f) \circ \Phi_{g}\right)=\left(\Phi_{g^{-1}}^{*} \mathbf{d} h\right)\left(B^{\sharp}(\mathbf{d} f)\right) \circ \Phi_{g}= \\
& =B\left(\mathbf{d} f, \Phi_{g^{-1}}^{*} \mathbf{d} h\right) \circ \Phi_{g}=\left\{f, \Phi_{g^{-1}}^{*} h \circ \Phi_{g}=\left\{\Phi_{g}^{*} f, h\right\}=\right. \\
& =\{f, h\}=\left(B^{\sharp}(\mathbf{d} f)\right)(\mathbf{d} h) .
\end{aligned}
$$

Thus, $B^{\sharp}(\mathbf{d} f)$ is a $G$-invariant vector field on $M$ and hence its value at $m \in S$ is tangent to $S$ (as stated above, it is tangent to the connected component of the isotropy type manifold through $m$, which is a submanifold of $S$ ). Hence, for all $\mathbf{d} f(m) \in\left((\mathfrak{g} \cdot m)^{\circ}\right)^{G_{m}}, f \in C^{\infty}(M)^{G}$, we have $B^{\sharp}(\mathbf{d} f(m)) \in T_{m} S$. 
To get a feeling for the condition in Theorem 3.2, we reprove directly that there is a reduced Poisson structure on every stratum of $\bar{M}$.

THEOREM 3.4. Let $(M,\{\cdot, \cdot\})$ be a smooth Poisson manifold and $G$ a Lie group acting canonically and properly on it. Each stratum $\bar{P}$ of $\bar{M}=M / G$ is a smooth Poisson manifold with Poisson bracket $\{\cdot, \cdot\}_{\bar{P}}$ given by

$$
\pi_{P}^{*}\left\{f_{\bar{P}}, h_{\bar{P}}\right\}_{\bar{P}}=\iota_{P}^{*}\left\{\pi^{*} \bar{f}, \pi^{*} \bar{h}\right\} \quad \text { for all } f_{\bar{P}}, h_{\bar{P}} \in C^{\infty}(\bar{P}),
$$

where $\bar{f}, \bar{h} \in C^{\infty}(\bar{M})$ are smooth extensions of $f_{\bar{P}}, h_{\bar{P}}$.

Proof. We have to show that the bracket $\{\cdot, \cdot\}_{\bar{P}}$ is well-defined and does not depend on the choice of the smooth extensions. Let $f_{\bar{P}}, h_{\bar{P}} \in C^{\infty}(\bar{P})$ be defined on an open set $U \subseteq \bar{P}$. By symmetry, it suffices to show that if $\bar{f}$ and $\bar{f}^{\prime}$ are two extensions of $f_{\bar{P}}$ and $\bar{h}$ an extension of $h_{\bar{P}}$, then

$$
\iota_{P}^{*}\left\{\pi^{*} \bar{f}, \pi^{*} \bar{h}\right\}=\iota_{P}^{*}\left\{\pi^{*} \bar{f}^{\prime}, \pi^{*} \bar{h}\right\} .
$$

Let $V \subseteq \bar{M}$ be the domain of definition of $\bar{f}, \bar{f}^{\prime}, \bar{h}$. We have

$$
\left\{\pi^{*} \bar{f}-\pi^{*} \bar{f}^{\prime}, \pi^{*} \bar{h}\right\}=-B^{\sharp}\left(\pi^{*} \mathbf{d} \bar{h}\right)\left(\pi^{*} \mathbf{d}\left(\bar{f}-\bar{f}^{\prime}\right)\right)=-\pi^{*} \mathbf{d}\left(\bar{f}-\bar{f}^{\prime}\right)\left(B^{\sharp}\left(\pi^{*} \mathbf{d} \bar{h}\right)\right) .
$$

We have seen in the proof of the preceding proposition that $B^{\sharp}\left(\pi^{*} \mathbf{d} \bar{h}\right)$ is a $G$-invariant vector field on $M$ and is thus tangent to the isotropy type manifolds (see [4, Section 3.5]). Hence, if $P$ is the union of strata of $M$ projecting to the stratum $\bar{P}$ of $\bar{M}$, the value of $B^{\sharp}\left(\pi^{*} \mathbf{d} \bar{h}\right)$ at $m$ is tangent to $P$ for all $m \in \pi^{-1}(V) \cap P=$ $\pi^{-1}(U)$. Since $\bar{f}-\bar{f}^{\prime}$ vanishes on $\bar{P}$, the differential $\pi^{*} \mathbf{d}\left(\bar{f}-\bar{f}^{\prime}\right)$ is zero on $T P$. Thus, we have $\left(\pi^{*} \mathbf{d}\left(\bar{f}-\bar{f}^{\prime}\right)\right)\left(B^{\sharp}\left(\pi^{*} \mathbf{d} \bar{h}\right)\right)(m)=0$ and hence $\left\{\pi^{*} \bar{f}-\pi^{*} \bar{f}^{\prime}, \pi^{*} \bar{h}\right\}(m)=$ 0 . This yields $\iota_{P}^{*}\left\{\pi^{*} \bar{f}-\pi^{*} \bar{f}^{\prime}, \pi^{*} \bar{h}\right\}=0$ and hence the required equality.

The Leibniz rule and Jacobi identity follow from the properties of $(M,\{\cdot, \cdot\})$.

\section{Acknowledgement}

We thank the referee for several remarks that have improved the exposition.

\section{References}

1. Falceto, F., Zambon, M.: An extension of the Marsden-Ratiu reduction for Poisson manifolds (2008). arXiv:0806.0638

2. Jotz, M., Ratiu, T.S., Sniatycki, J.: Singular Dirac reduction (2009). arXiv:0901.3062

3. Marsden, J.E., Ratiu, T.S.: Reduction of Poisson manifolds. Lett. Math. Phys. 11(2), 161-169 (1986) (ISSN 0377-9017)

4. Ortega, J.-P., Ratiu, T.S.: Momentum maps and Hamiltonian reduction. Progress in Mathematics (Boston, Mass.), vol. 222, xxxiv, 497 p. Birkhäuser, Boston (2004)

5. Ortega, J.-P., Ratiu, T.S.: Singular reduction of Poisson manifolds. Lett. Math. Phys. 46(4), 359-372 (1998) (ISSN 0377-9017) 